\title{
Fuzzy-marker-based segmentation using hierarchies
}

\author{
Gabriel Barbosa da Fonseca ${ }^{1,2}$, Benjamin Perret ${ }^{2}$, Romain Negrel $^{3}$, Jean \\ Cousty $^{2}$, and Silvio Jamil Ferzoli Guimarães ${ }^{1}$ \\ 1 Laboratory of Image and Multimedia Data Science \\ Pontifical Catholic University of Minas Gerais, Brazil, 31980-110 \\ sjamil@pucminas.br \\ 2 LIGM, Université Gustave Eiffel, CNRS, ESIEE Paris, F-77454 Marne-la-Vallée \\ \{gabriel.fonseca, benjamin.perret, jean.cousty\}@esiee.fr \\ 3 ESIEE Paris, Université Gustave Eiffel \\ romain.negrel@esiee.fr
}

\begin{abstract}
This article extends a classical marker-based image segmentation method proposed by Salembier and Garrido in 2000. In the original approach, the segmentation relies on two sets of pixels which play the role of object and background markers. In the proposed extension, the markers are not represented by crisp sets, but by fuzzy ones, i.e., functions of the image domain into the real interval $[0,1]$ indicating the degree of membership of each pixel to the markers. We show that when the fuzzy markers are indicator functions of crisp sets, the proposed method produces the same result as the original one. We present a linear-time algorithm for computing the result of the proposed method given two fuzzy markers and we establish the correctness of this algorithm. Additionally, we discuss possible applications of the proposed approach, such as adjusting marker strength in interactive image segmentation procedures and optimizing marker locations with gradient descent methods.
\end{abstract}

\section{Introduction}

Image segmentation is one of the fundamental tasks in image processing, and can be described as partitioning an image into distinct regions that comprise similar objects. Many argue that image segmentation is in fact a multi-scale problem, where regions at a coarser detail level are formed by a merging of regions at a finer detail level [11125]. Following this idea, authors often utilize hierarchies constructed from a given image to produce a segmentation [1]420|23, and efficient algorithms for constructing such hierarchies have been proposed [16].

The authors thank Conselho Nacional de Desenvolvimento Científico e Tecnológico CNPq - (PQ 310075/2019-0), Coordenação de Aperfeiçoamento de Pessoal de Nível Superior - CAPES - (Grant COFECUB 88887.191730/2018-00) and Fundação de Amparo à Pesquisa do Estado de Minas Gerais - FAPEMIG - (Grants PPM-0000618). 


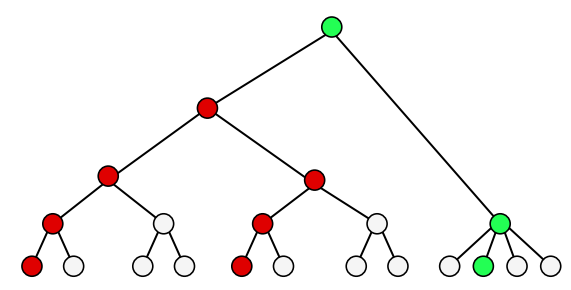

(a)

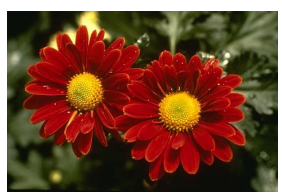

(b)

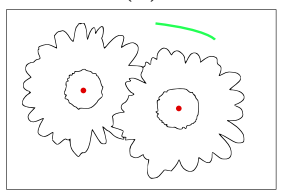

(c)

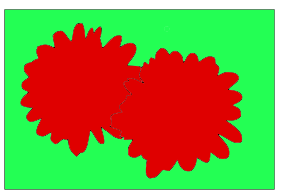

(d)

Fig. 1: A tree representation for a marker-based image segmentation with respect to a hierarchy on (a). On (b), the original image, on (c) a representation of the partitions with markers, and a segmentation on $(d)$.

Even with the recent advances in the area of image processing, automatic segmentation can still be a very challenging task. The introduction of prior knowledge in the form of markers (often called seeds) that carry information about the location of objects of interest can drastically improve segmentation results. Many works study different approaches for producing marker-based segmentations, ranging from the classic graph-based works relying on watersheds [7/23], graph cuts [195], random walks [12, geodesics [2] and shortest paths [10], to the more recent works based on convolutional neural networks [13:24,14]. Also, when markers are provided by users over an interaction system, they can take different shapes. These shapes include scribbles [2712, bounding boxes [19], points 13, and image regions 2015. In classical graph-based methods, markers usually have a binary nature, i.e., a region of the image is either marked and represents an object with total certainty, or is unmarked. With that, distinct markers in the same image guide the segmentation with equal power.

Different approaches were proposed for controlling the way markers propagate when guiding a segmentation. In [23], the shape of the surface where markers propagate is modified to simulate a flooding by a viscous fluid, but different markers still guide the segmentation with equal power. In [6], the costs for propagating a marker to a region are dynamically estimated during propagation time. In this work, we are interested in the scenario where markers have a fuzzy nature instead of a binary one, and where different markers propagate and guide a segmentation with different power.

For that, we propose an extension of a classical method introduced in [20], which uses two sets of pixels as object and background markers and propagate them over a hierarchy of partitions to produce a two-class segmentation, i.e., a partition of the image into a region classified as object and a region classified 
as background. In this extension, markers are not binary, but represented by positive real functions, referred to as fuzzy markers. The value of each element in a fuzzy marker represents its membership degree to the marker and dictates the influence of the marker at its location.

To present our extension, we rely on a characterization of 20] based on connection values (also called "fuzzy connectedness" [22] or "degree of connectivity" [18]). In our extension, we produce a segmentation based on fuzzy connection values to the object and background markers, similarly to the method proposed in 21]. However, contrary to [21], the fuzzy connection values proposed in this work also take the membership degree of elements to fuzzy markers into consideration.

The main contributions of this article are fourfold. First, we propose an extension of a classical segmentation method, where markers are fuzzy instead of being crisp. Second, we show that this method is indeed equivalent to the original one when markers are crisp (with Property 5). Third, we provide an efficient algorithm for computing in linear-time the proposed segmentation. Finally, we establish the correctness of the proposed algorithm (with Corollary 9). Due to space limitation, proofs of the presented properties will be included in an forthcoming extended version of this article. Furthermore, we discuss possible applications of the proposed method, such as adjusting the strength of markers when performing interactive segmentation and optimizing seed locations using gradient descent methods.

This article is organized as follows. In Section 2, we present the marker-based segmentation proposed in [20. In Section 3, we define an extension of the markerbased segmentation, where markers are represented by fuzzy sets. We introduce an efficient algorithm for computing the fuzzy-marker-based segmentation in Section 4. Finally, in Section 5 we present the conclusion, possible applications of the proposed method, and directions for future works.

\section{Marker-based segmentation using hierarchies}

In this section, we present a classical approach for performing a marker-based image segmentation proposed by Salembier and Garrido in 20. This method relies on extracting relevant regions of a hierarchy with respect to provided markers. First, we provide fundamental notions to define indexed hierarchies. Then, we present the notion of a marker-based segmentation from a hierarchy as introduced in 20. Finally, we present a characterization of this segmentation based on ultrametric distances associated to given hierarchies. This characterization allows us to extend in Section 3 the method proposed in [20] to the case where the markers are fuzzy instead of being crisp.

In this article, the symbol $V$ denotes a finite nonempty set. A hierarchy $\mathcal{H}$ (on $V$ ) is a set of subsets of $V$ such that:

1. $V$ is an element of $\mathcal{H}$;

2. for every element $x$ of $V$, the singleton $\{x\}$ belongs to $\mathcal{H}$; and 
3. for any two elements $X$ and $Y$ of $\mathcal{H}$ if the intersection of $X$ and $Y$ is nonempty, then $X$ either includes $Y$ or is included in $Y$.

Let $\mathcal{H}$ be any hierarchy, any element of $\mathcal{H}$ is called a region of $\mathcal{H}$. For any region $X$ of $\mathcal{H}$, we define the index of $X$ as a positive real value denoted by $\omega(X)$. An indexed hierarchy (on $V$ ) is a pair $(\mathcal{H}, \omega)$, where $\mathcal{H}$ is a hierarchy and where $\omega$ is a function from $\mathcal{H}$ to $\mathbb{R}^{+}$such that:

1. $\omega(X)=0$ if and only if $X$ is a singleton; and

2. for any two regions $X$ and $Y$ of $\mathcal{H}$, if $X$ is included in $Y$, then we have $\omega(X)<\omega(Y)$.

Let $\mathcal{H}$ be a hierarchy and let $X$ and $Y$ be two regions of $\mathcal{H}$. The region $X$ is a parent of $Y$ and $Y$ is a child of $X$ if $Y$ is included in $X$ and if any region of $\mathcal{H}$ which is proper superset of $Y$ is also a superset of $X$. A region $R$ of $\mathcal{H}$ is called a leaf (resp. root) of $\mathcal{H}$ if it is not the parent (resp. child) of any region of $\mathcal{H}$. It can be observed that $V$ is the only root of $\mathcal{H}$ and that the set of leaves of $\mathcal{H}$ is precisely the set of all singletons on $V$. It can also be noticed that any non-root region $X$ of $\mathcal{H}$ has a unique parent, which is denoted by $\operatorname{par}(X)$ in the following. A hierarchy $\mathcal{H}$ is considered a binary hierarchy if any non-leaf region has exactly two distinct children.

In the remaining part of this article, the pair $(\mathcal{H}, \omega)$ denotes an indexed binary hierarchy, that is an indexed hierarchy such that $\mathcal{H}$ is a binary hierarchy.

Let us now provide the definition of the method introduced in 20]. The main idea is to produce a partition by classifying as object any region of the hierarchy that intersects a given object marker and that does not intersect the background marker.

Let $O$ and $B$ be two subsets of $V$. The marker-based segmentation (of $V$ ) for $(O, B)$ (with respect to $(\mathcal{H}, \omega)$ ), denoted by $S_{\mathcal{H}}(O, B)$ is the union of the regions of $\mathcal{H}$ which contain an element of $O$ but no element of $B$ :

$$
S_{\mathcal{H}}(O, B)=\cup\{X \in \mathcal{H} \mid X \cap O \neq \emptyset, X \cap B=\emptyset\} .
$$

Our main interest in this article is to extend the notion of a segmentation for a pair of subsets of the space called markers to the case where the markers are fuzzy. In order to present this extension, we will rely on an alternative characterization of the marker-based segmentation based on a (ultrametric) distance induced by the hierarchy.

We define $d_{\mathcal{H}}$ as the function from $V \times V$ to $\mathbb{R}^{+}$such that, for any two elements $x$ and $y$ of $V$, the value $d_{\mathcal{H}}(x, y)$ is the index of the smallest region of $\mathcal{H}$ which contains both $x$ and $y$ :

$$
d_{\mathcal{H}}(x, y)=\min \{\omega(X) \mid X \in \mathcal{H}, x \in X, y \in X\} .
$$

It is well known that the function $d_{\mathcal{H}}$ is a distance which is furthermore ultrametric [3]. Let $x$ be any element of $V$ and let $Y$ be any subset of $V$. We define the connection value of $x$ to $Y$ (for the hierarchy $\mathcal{H}$ ), denoted by $\mathcal{C}_{\mathcal{H}}(x, Y)$, as the shortest distance between $x$ and an element of $Y$ :

$$
\mathcal{C}_{\mathcal{H}}(x, Y)=\min \left\{d_{\mathcal{H}}(x, y) \mid y \in Y\right\} .
$$


Property 1. Let $O$ and $B$ be two subsets of $V$. The following statement holds true:

$$
S_{\mathcal{H}}(O, B)=\left\{x \in V \mid \mathcal{C}_{\mathcal{H}}(x, O)<\mathcal{C}_{\mathcal{H}}(x, B)\right\} .
$$

In other words, Property 1 states that a point $x$ belongs to the marker-based segmentation $S_{\mathcal{H}}(O, B)$ if the shortest distance between $x$ and an element of $O$ is smaller than the shortest distance between $x$ and an element of $B$.

\section{$3 \quad$ Fuzzy-marker-based segmentation}

The marker-based segmentation defined in [20] and presented in Section 2 , relies on two subsets which play the role of markers. In this section we define an extension in which markers are not crisp sets anymore but fuzzy sets.

Fuzzy sets can be seen as membership functions defined on a set, in which each element has a membership degree. On a crisp set, the relationship between an element and a set is binary, i.e., either the element belongs to the set or not. With a fuzzy set, elements can be considered partially included in a set, with higher membership degrees meaning a "stronger" inclusion in the set.

A fuzzy set (on $V$ ) is defined as a function from $V$ to the real interval $[0,1]$. Let $\mu$ be a fuzzy set. The function $\mu$ is also referred to as a membership function of $V$, where for any element $x$ of $V$, the value $\mu(x)$ represents the membership degree of $x$ in $\mu$.

In the remaining of this article, fuzzy markers will be represented by fuzzy sets. We now extend the notion of connection value to fuzzy sets.

Definition 2. (Fuzzy connection value) Let $x$ be an element of $V$, and let $\mu$ be a fuzzy set. The fuzzy connection value of $x$ to $\mu$ (for $(\mathcal{H}, \omega)$ ), denoted by $\mathcal{C}_{\mathcal{H}}^{f}(x, \mu)$, is defined by:

$$
\mathcal{C}_{\mathcal{H}}^{f}(x, \mu)=\min \left\{\alpha(\mu(y))\left(1-\mu(y)+d_{\mathcal{H}}(x, y)\right) \mid y \in V\right\},
$$

where $\alpha$ is a decreasing function such that $\alpha(1)=1$ and $\alpha(0)$ is strictly greater than the maximal value of $d_{\mathcal{H}}$, i.e., $\alpha(0)>\max \left\{d_{\mathcal{H}}(x, y) \mid x \in V, y \in V\right\}$.

The notion of a fuzzy connection value is an extension of the one of connection value. The information given by the membership degree of an element to a fuzzy set is incorporated to the fuzzy connection value by the term $\alpha(\mu(y))$. Observe that for two elements $x$ and $y$ in $V$, such that $\mu(y)$ is equal to 1 , we have that $\alpha(\mu(y))\left(1-\mu(y)+d_{\mathcal{H}}(x, y)\right)$ is equal to $d_{\mathcal{H}}(x, y)$. Since $\alpha$ is a decreasing function, as the value $\mu(y)$ decreases, the overall fuzzy connection value increases. In other words, we want that elements with low membership degrees lead to greater fuzzy connection values than elements with high membership degrees. The term $1-\mu(y)$ and the constraint on $\alpha(0)$ allow us to show the link between connection values and fuzzy connection values, which is formally introduced in Property 3.

The following property makes the link between connection values and fuzzy connection values explicit by means of indicator functions. 


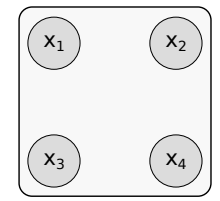

(a) $V$

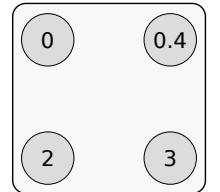

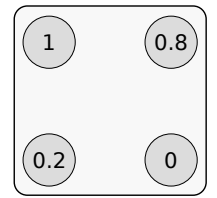

(b) $\mu_{O}$

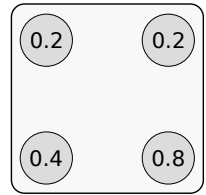

(c) $\mu_{B}$

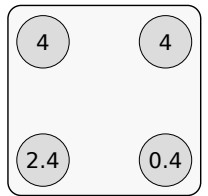

(e) Connection values to $\mu_{O}$ (f) Connection values to $\mu_{B}$

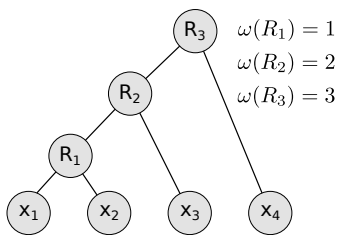

(d) $(\mathcal{H}, \omega)$

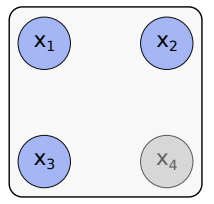

(g) $S_{\mathcal{H}}^{f}\left(\mu_{O}, \mu_{B}\right)$

Fig. 2: Example of a fuzzy-marker-based segmentation. Graphical representations of: (a) a set $V$; (b,c) fuzzy sets $\mu_{O}$ and $\mu_{B} ;(\mathrm{d})$ an indexed hierarchy $(\mathcal{H}, \omega)$ on $V$; (e,f) fuzzy connection values to $\mu_{O}$ and $\mu_{B}$, respectively; (g) the segmentation $S_{\mathcal{H}}^{f}\left(\mu_{O}, \mu_{B}\right)$ highlighted in blue. The $\alpha$ function is given by $\alpha(x)=(6-5 x)$.

Let $A$ be a subset of $V$. The indicator function of $A$ is the fuzzy set $\mathbb{1}_{A}$, such that for any $x$ in $V$, the value $\mathbb{1}_{A}$ is equal to 1 if $x$ belongs to $A$ and is equal to 0 otherwise.

Property 3. Let $A$ be a non-empty subset of $V$, and $\mathbb{1}_{A}$ be the indicator function of $A$. Then, the following statement holds true:

$$
\forall x \in V, \quad \mathcal{C}_{\mathcal{H}}(x, A)=\mathcal{C}_{\mathcal{H}}^{f}\left(x, \mathbb{1}_{A}\right) .
$$

We are now ready to extend the notion of a segmentation of a set $V$ for a pair of subsets of $V$ to a segmentation of $V$ for a pair of fuzzy sets of $V$.

Definition 4. (Fuzzy-marker-based segmentation) Let $\mu_{O}$ and $\mu_{B}$ be two fuzzy sets. The fuzzy-marker-based segmentation (of $V$ ) for $\left(\mu_{O}, \mu_{B}\right)$ (with respect to $(\mathcal{H}, \omega))$, denoted by $S_{\mathcal{H}}^{f}\left(\mu_{O}, \mu_{B}\right)$ is defined by

$$
S_{\mathcal{H}}^{f}\left(\mu_{O}, \mu_{B}\right)=\left\{x \in V \mid \mathcal{C}_{\mathcal{H}}^{f}\left(x, \mu_{O}\right)<\mathcal{C}_{\mathcal{H}}^{f}\left(x, \mu_{B}\right)\right\}
$$

In other words, the fuzzy-marker-based segmentation of $V$ for $\left(\mu_{O}, \mu_{B}\right)$ is the set that contains every element of $V$ with a fuzzy connection value to $\mu_{O}$ smaller than to $\mu_{B}$.

An example of a fuzzy-marker-based segmentation for a pair of fuzzy sets is illustrated in Figure 2 A set $V$ is illustrated in Figure 2a, followed by the fuzzy markers $\mu_{O}$ and $\mu_{B}$ in Figures 2b and 2c, respectively. We can observe in Figure $2 \mathrm{~d}$ a tree representation of a given indexed hierarchy. The fuzzy connection values of the elements of $V$ to the fuzzy sets $\mu_{O}$ and $\mu_{B}$ can be observed in Figures $2 \mathrm{e}$ and $2 \mathrm{f}$ respectively. For the elements $x_{1}, x_{2}, x_{3}$, the fuzzy connection 
value to the fuzzy set $\mu_{O}$ is smaller than to the fuzzy set $\mu_{B}$. Consequently, $\left\{x_{1}, x_{2}, x_{3}\right\}$ is the segmentation $S_{\mathcal{H}}^{f}\left(\mu_{O}, \mu_{B}\right)$, as shown in Figure $2 \mathrm{~g}$.

The following property shows the relationship between the fuzzy-markerbased segmentation proposed in this article and the marker-based segmentation proposed in 20.

Property 5. Let $O$ and $B$ be two subsets of $V$, and $\mathbb{1}_{O}$ and $\mathbb{1}_{B}$ be the indicator functions of $O$ and $B$, respectively. Then, the following statement holds true:

$$
S_{\mathcal{H}}^{f}\left(\mathbb{1}_{O}, \mathbb{1}_{B}\right)=S_{\mathcal{H}}(O, B) .
$$

In other words, Property 5 states that when the fuzzy markers indeed represent crisp sets, the marker-based segmentation is the same as the fuzzy-markerbased segmentation.

\section{Efficient computation of fuzzy-marker-based segmentation}

In this section, we present an efficient algorithm for computing the fuzzy connection values for every element of a set to a given fuzzy set on this space. Before presenting the algorithm, we give some definitions and properties that allow us to introduce the algorithm and prove its correctness. Finally, we give a brief discussion about the complexity of the proposed algorithm.

Let $X$ and $Y$ be two distinct regions of $\mathcal{H}$. The region $X$ is a sibling of $Y$ and $X$ and $Y$ are siblings if the parent of $X$ and the parent of $Y$ are the same. Since $\mathcal{H}$ is a binary hierarchy, every region on $\mathcal{H}$ that has a parent has exactly one sibling. If $X$ is a non-root region of $\mathcal{H}$, we denote by $\operatorname{sib}(X)$ the unique sibling of $X$.

We define the restriction of a fuzzy set $\mu$ to a subset $X$ of $V$, denoted by $\mu \downarrow_{X}$, as the fuzzy set such that $\mu \downarrow_{X}(x)=\mu(x)$ for any $x$ in $X$, and $\mu_{\downarrow_{X}}(x)=0$ for any $x$ in $V \backslash X$, i.e., $\mu \downarrow_{X}=\inf \left(\mu, \mathbb{1}_{X}\right)$.

Property 6. Let $\mu$ be a fuzzy set and let $x$ be an element in $V$. Then, the fuzzy connection value from $x$ to $\mu$ is given by:

$$
\mathcal{C}_{\mathcal{H}}^{f}(x, \mu)=\min \left\{\mathcal{C}_{\mathcal{H}}^{f}\left(x, \mu \downarrow_{V \backslash\{x\}}\right), \alpha(\mu(x))(1-\mu(x))\right\} .
$$

From Property 6, we see that the connection value of $x$ to $\mu$ can be obtained from the connection value of $x$ to the restriction of $\mu$ to $V \backslash\{x\}$ and from $\alpha(\mu(x))(1-\mu(x))$. The computation of the later term can be done in constant time. We will now present an efficient way to compute the former one.

Let $\mu$ be a fuzzy set. We denote by $\max (\mu)$ the maximum of $\mu(x)$ for all $x$ in $V$. 


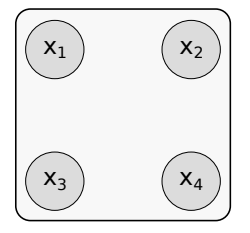

(a) $V=\left\{x_{1}, x_{2}, x_{3}, x_{4}\right\}$

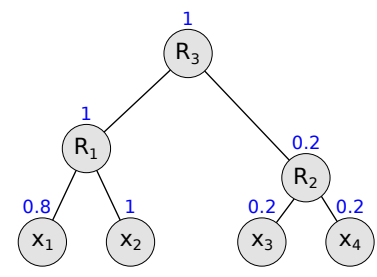

(d) $\max \left(\mu \downarrow_{R}\right)$

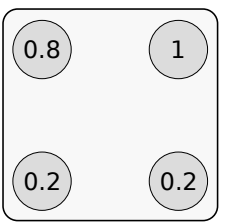

(b) Fuzzy set $\mu$

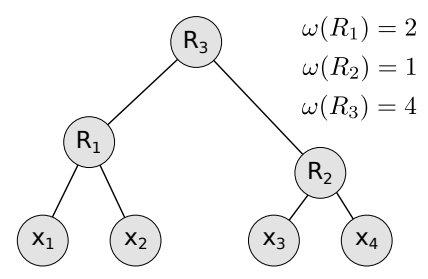

(c) $(\mathcal{H}, \omega)$

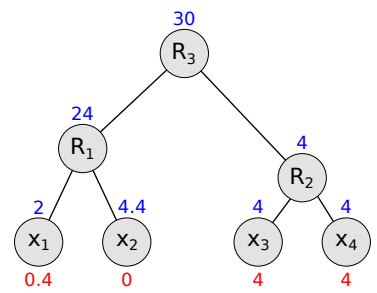

(e) Connection values

Fig. 3: A set $V$ on (a). A fuzzy set $\mu$ on (b). A representation of an indexed hierarchy $(\mathcal{H}, \omega)$ on $(\mathrm{c})$. On $(\mathrm{d})$, the value $\max \left(\mu \downarrow_{R}\right)$ for each region of $\mathcal{H}$ in blue. On (e), the regional connection value of each region of $\mathcal{H}$ in blue and the fuzzy connection value of each element of $V$ (the leaves) in red. For this example, we set $\alpha(x)=6-5 x$.

Definition 7. (Regional connection value) Let $\mu$ be a fuzzy set and let $R$ be a region of $\mathcal{H}$. The regional connection value of $R$, denoted by $T_{\mathcal{H}}^{\mu}(R)$, is defined by:

$T_{\mathcal{H}}^{\mu}(R)=\alpha(0)(1+\omega(R))$, if $R=V$ (i.e., $R$ is the root of $\left.\mathcal{H}\right)$; and

$T_{\mathcal{H}}^{\mu}(R)=\min \left(T_{\mathcal{H}}^{\mu}(\operatorname{par}(R)), \alpha\left(\max \left(\mu \downarrow_{s i b(R)}\right)\right)\left(1-\max \left(\mu \downarrow_{s i b(R)}\right)+\omega(\operatorname{par}(R))\right)\right)$,

$$
\text { if } R \neq V \text {. }
$$

The regional connection value $T_{\mathcal{H}}^{\mu}(R)$ of each region can be obtained by browsing the hierarchy $\mathcal{H}$ in root-to-leaves order, i.e., an order in which a parent region is processed before its children. Algorithm 1 presents the different steps to compute these values in linear time with respect to the number of regions in $\mathcal{H}$.

In Figure 3 we have illustrations of a set $V$ in Figure 3a, a fuzzy set $\mu$ in Figure $3 \mathrm{~b}$, and an indexed hierarchy $(\mathcal{H}, \omega)$ in Figure $3 \mathrm{c}$. In Figure $3 \mathrm{e}$, we have the values of the regional connection values of every region of the hierarchy in blue. For example, by using the equations from Definition 7, the regional connection value of the root of $\mathcal{H}$ (region $R_{3}$ ) is given by $\alpha(0)\left(1+\omega\left(R_{3}\right)\right.$ ). By setting $\alpha=6-5 x$, as in the example illustrated in Figure 3 , we have that $T_{\mathcal{H}}^{\mu}\left(R_{3}\right)=(6)(1+4)=30$. For the left child of the root (region $\left.R_{1}\right)$, its regional connection value is given by the minimum between the values $T_{\mathcal{H}}^{\mu}\left(R_{3}\right)$ and $\alpha\left(\max \left(\mu \downarrow_{R_{2}}\right)\right)\left(1-\max \left(\mu \downarrow_{R_{2}}\right)+\omega\left(R_{3}\right)\right)=(6-5 * 0.2)(1-0.2+4)=24$. As the value $T_{\mathcal{H}}^{\mu}\left(R_{3}\right)=30$, we have $T_{\mathcal{H}}^{\mu}\left(R_{1}\right)=\min (30,24)=24$. 
As we will see with Property 8 and Corollary 9, the regional connection values allows us to compute the fuzzy connection values of every element $x$ in $V$. With the proposed algorithm, we can efficiently compute the fuzzy connection values from all elements of a set to two distinct fuzzy sets. Having the fuzzy connection values to a pair of fuzzy sets, one can compute the fuzzy-markerbased segmentation of a given set with Definition 4

The following property links the regional connection value to fuzzy connection values. This property allows us to show the correctness of the proposed algorithm for computing fuzzy connection values.

Property 8. Let $\mu$ be a fuzzy set and let $x$ be an element of $V$. Then, the fuzzy connection value of $x$ to the restriction of $\mu$ to $V \backslash\{x\}$ is equal to $T_{\mathcal{H}}^{\mu}(\{x\})$ :

$$
\mathcal{C}_{\mathcal{H}}^{f}\left(x, \mu \downarrow_{V \backslash\{x\}}\right)=T_{\mathcal{H}}^{\mu}(\{x\}) .
$$

Then, from Properties 6 and 8 we can derive Corollary 9 , which presents how to obtain the fuzzy connection values for all $x$ in $V$ to $\mu$ by using the regional connection values.

Corollary 9. Let $x$ be an element of $V$, and $\mu$ be a fuzzy set. For any $x$ in $V$, the fuzzy connection value $\mathcal{C}_{\mathcal{H}}^{f}(x, \mu)$ is given by:

$$
\mathcal{C}_{\mathcal{H}}^{f}(x, \mu)=\min \left\{T_{\mathcal{H}}^{\mu}(\{x\}), \alpha(\mu(x))(1-\mu(x))\right\}
$$

A set $V$ is illustrated in Figure 3 a followed by a fuzzy set $\mu$ illustrated in Figure $3 \mathrm{~b}$ and a hierarchy $(\mathcal{H}, \omega)$ illustrated in Figure $3 \mathrm{c}$. In Figure $3 \mathrm{e}$, we can observe the fuzzy connection values (in red) of the elements of the set $V$ to $\mu$ with respect to $(\mathcal{H}, \omega)$. By observing Figure $3 \mathrm{e}$ and using the equation from Corollary 9 , it can be seen that the fuzzy connection value of the leaf $x_{1}$ is equal to the minimum between $T_{\mathcal{H}}^{\mu}\left(\left\{x_{1}\right\}\right)$ and $\alpha\left(\mu\left(x_{1}\right)\right)\left(1-\mu\left(x_{1}\right)\right)$. By setting $\alpha(x)=6-5 x$, we have that $T_{\mathcal{H}}^{\mu}\left(\left\{x_{1}\right\}\right)=2$ and $\alpha(0.8)(1-0.8)=0.4$, thus the fuzzy connection value of $x_{1}$ to $\mu$ is equal to 0.4 .

The computation of the value $\max \left(\mu \downarrow_{R}\right)$ for a function $\mu$ and every regions $R$ in $\mathcal{H}$ can be done with a single pass on the hierarchy in leaves-toroot order, i.e., in which a region is processed after its children. The computation of $T_{\mathcal{H}}^{\mu}(\{x\})$ for every $x$ in $V$ is done with a single pass on the hierarchy, in linear time with respect to the number of regions of $\mathcal{H}$. Then, after computing $T_{\mathcal{H}}^{\mu}(\{x\})$ for every $x$ in $V$, computing the fuzzy connection values $\mathcal{C}_{\mathcal{H}}^{f}(x, \mu)$ for all $x$ in $V$ is done in linear time with respect to the number of elements in $V$, as it can be observed in Algorithm 2. As the number of re- 
gions in $\mathcal{H}$ is at most $2|V|-1$, this leads to an overall complexity of $O(|V|)$.

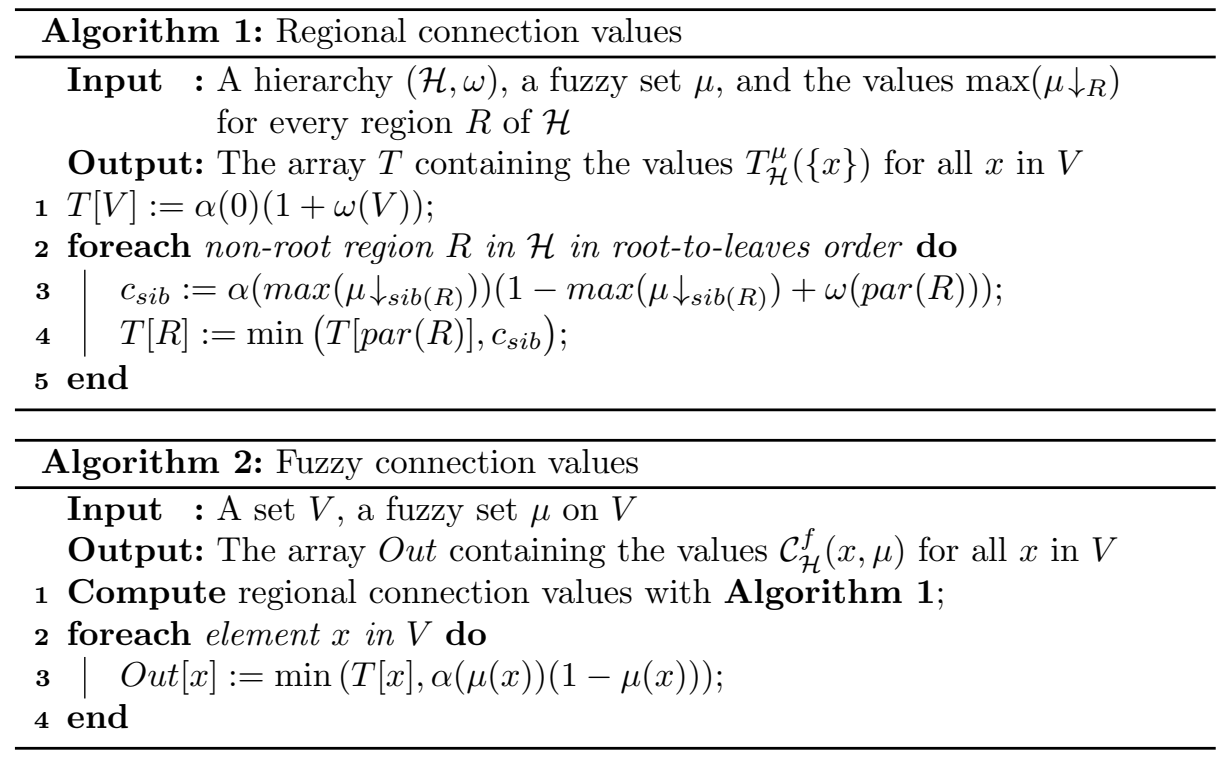

\section{Conclusion and discussions}

In this work, we propose an extension of a classical method of marker-based image segmentation to the case where markers are represented by fuzzy sets. We also propose an algorithm for computing the fuzzy-marker-based segmentation in linear time, and prove its correctness. With the proposed extension, a new extent of applications becomes possible, such as:

Interactive segmentation with adjustable marker strength: In an interactive segmentation framework, markers usually represent the location of objects of interest, and distinct markers guide the segmentation with equal power. By using the method proposed in this article, it is possible to add extra information to the markers, in the form of marker strength. With a fuzzy marker, we can adjust its membership degree from 0 to 1 , where 1 denotes the maximum strength, and 0 the minimum.

An example of such application is illustrated in Figure 4. The segmentations produced in the illustration are computed with a watershed by area hierarchy [8] created over a 4-adjacency graph weighted by a SED gradient 9 . We can see on the second row of Figure 4 that when the markers are crisp (with strength of 1 on all marker locations) there is a leakage of the object segmentation to the background. We show that we can decrease this leakage by adjusting the strength of the object markers, without the need of changing the locations of any markers. The code for performing a fuzzy-marker-based interactive segmentation with adjustable marker strength developed using the HIGRA library [17] is available at https://higra.readthedocs.io/en/latest/notebooks.html. 

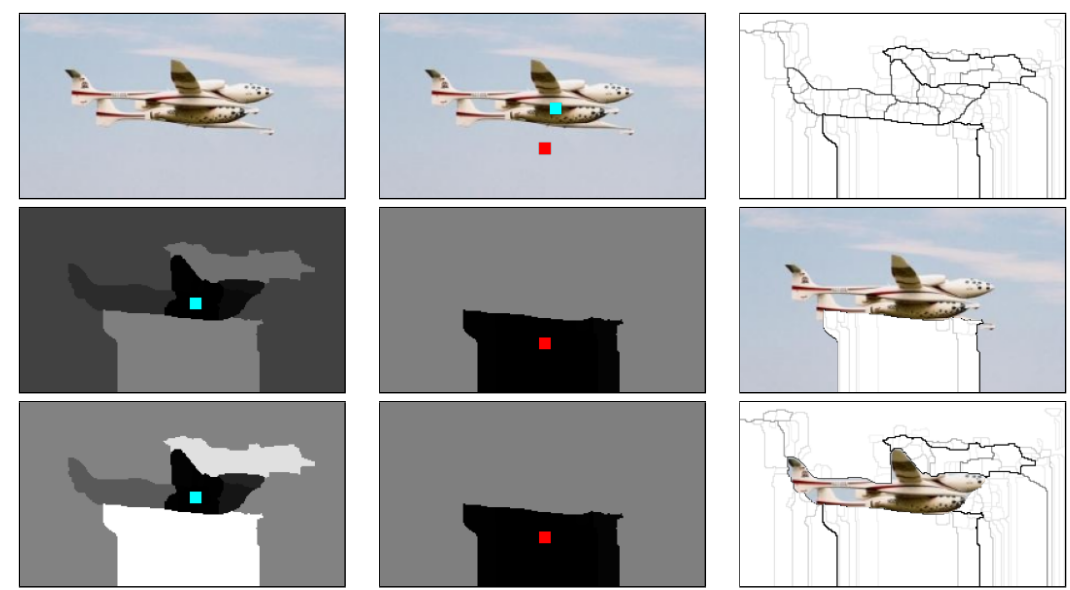

Fig. 4: Example of fuzzy-marker-based interactive segmentation. On the top row, from left to right: an image, object and background makers (in blue and red, respectively) superimposed over the image, and a saliency map representation of a hierarchy on the image. On the second and third row we can observe the map of fuzzy connection values from the regions of the image to the object marker, followed by the map of fuzzy connection values to the background marker, and finally the produced segmentation. On the maps of connection values, darker colors represent smaller values. On the second row, the markers are crisp, and on the third row, the value of the object markers is set to 0.5 .

Marker optimization: Given a ground-truth segmentation, we can also ask ourselves what are the optimal markers to obtain this segmentation. It can be seen that the function $\mathcal{C}_{\mathcal{H}}^{f}$ given in Definition 2 is sub-differentiable with respect to $\mu$, this allows us to use a classical gradient descent algorithm to optimize markers for a given ground-truth.

Given an image and a pair of fuzzy markers, we can produce a segmentation for the given markers. With the produced segmentation, we can compute a segmentation loss with respect to the ground-truth, using for instance a pixelwise cross-entropy loss. We search for a pair of markers that minimize the loss function, i.e., that produce a segmentation which is closer to the ground-truth. We can find a solution to this minimization problem with gradient descent, thus optimizing markers for a given ground-truth segmentation.

An example of a marker optimization is shown in Figure 5 . In the example, we use a pair of object and background markers, such that the background marker intersects the object of interest. Due to part of the background marker being located over the object, the produced segmentation has regions of the object wrongly classified as background. After the optimization process, we can observe that the produced markers lead to a better object segmentation, i.e., a segmentation which is more similar to the considered ground-truth. For the example in Figure 5 , we use a watershed by area hierarchy created over a 4- 


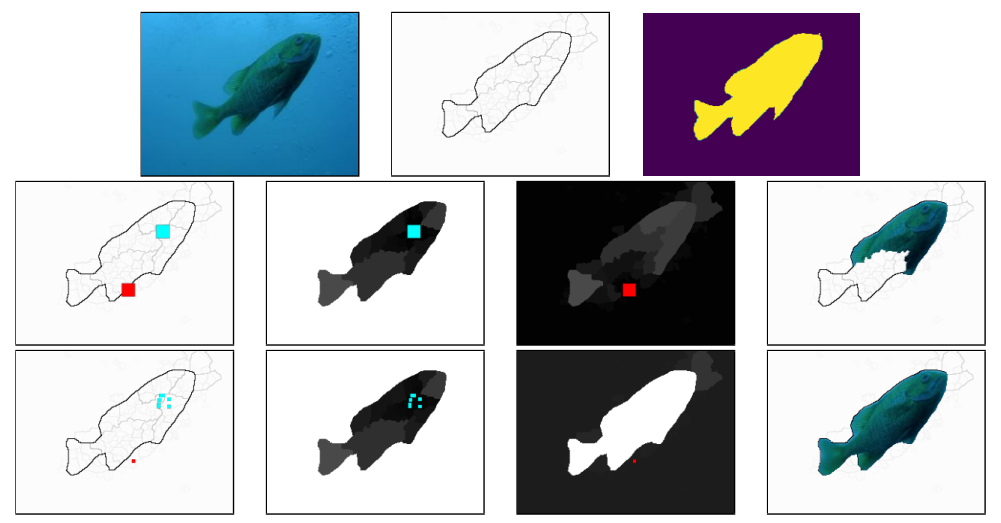

Fig. 5: Illustration of the marker optimization. On the top row, from left to right: an image; a representation of a saliency map of a hierarchy for the image; and the ground-truth of the object segmentation. On the second and third row: the markers (object in blue, background in red) over the saliency maps, followed by the map of fuzzy connection values to the object marker, the map of fuzzy connection values to the background marker, and the fuzzy-marker-based segmentation. On the second row we have the initial crisp markers, and on the bottom row we have the optimized markers. On the maps of fuzzy connection values, darker color represents lower values.

adjacency graph weighted by a SED gradient. During the optimization process we also introduce a set of regularization functions to reduce the size and increase the smoothness of the markers. Finally, we apply a grayscale dilation on the markers after the optimization for better visualization.

In future works, we will extend the studies on learning marker locations, using the proposed optimization framework to train a marker proposal convolutional neural network. Ideally, the markers proposed by the network would be able to recover the segmentation ground-truth, and follow shape constraints that characterize easily editable markers.

\section{References}

1. Arbeláez, P., Maire, M., Fowlkes, C., Malik, J.: Contour detection and hierarchical image segmentation. PAMI 33(5), 898-916 (2011)

2. Bai, X., Sapiro, G.: Geodesic matting: A framework for fast interactive image and video segmentation and matting. IJCV 82(2), 113-132 (2009)

3. Benzecri, J.P., et al.: L'analyse des données. 1. la taxinomie. Paris: Dunod 195, 196 (1973)

4. Beucher, S.: Watershed, hierarchical segmentation and waterfall algorithm. In: ISMM, pp. 69-76. Springer (1994)

5. Boykov, Y.Y., Jolly, M.P.: Interactive graph cuts for optimal boundary \& region segmentation of objects in nd images. In: ICCV. vol. 1, pp. 105-112. IEEE (2001) 
6. Bragantini, J., Martins, S.B., Castelo-Fernandez, C., Falcão, A.X.: Graph-based image segmentation using dynamic trees. In: CIARP. pp. 470-478. Springer (2018)

7. Cousty, J., Bertrand, G., Najman, L., Couprie, M.: Watershed cuts: Minimum spanning forests and the drop of water principle. PAMI 31(8), 1362-1374 (2008)

8. Cousty, J., Najman, L.: Incremental algorithm for hierarchical minimum spanning forests and saliency of watershed cuts. In: ISMM. pp. 272-283. Springer (2011)

9. Dollár, P., Zitnick, C.L.: Fast edge detection using structured forests. PAMI 37(8), 1558-1570 (2014)

10. Falcão, A.X., Stolfi, J., de Alencar Lotufo, R.: The image foresting transform: Theory, algorithms, and applications. PAMI 26(1), 19-29 (2004)

11. Gómez, D., Yanez, J., Guada, C., Rodríguez, J.T., Montero, J., Zarrazola, E.: Fuzzy image segmentation based upon hierarchical clustering. Knowledge-Based Systems 87, 26-37 (2015)

12. Grady, L.: Random walks for image segmentation. PAMI 28(11), 1768-1783 (2006)

13. Jang, W.D., Kim, C.S.: Interactive image segmentation via backpropagating refinement scheme. In: CVPR. pp. 5297-5306. IEEE (2019)

14. Li, Z., Chen, Q., Koltun, V.: Interactive image segmentation with latent diversity. In: CVPR. pp. 577-585. IEEE (2018)

15. Malmberg, F., Nordenskjöld, R., Strand, R., Kullberg, J.: Smartpaint: a tool for interactive segmentation of medical volume images. CMBBE 5(1), 36-44 (2017)

16. Najman, L., Cousty, J., Perret, B.: Playing with kruskal: algorithms for morphological trees in edge-weighted graphs. In: ISMM. pp. 135-146. Springer (2013)

17. Perret, B., Chierchia, G., Cousty, J., Guimarães, S.J.F., Kenmochi, Y., Najman, L.: Higra: Hierarchical graph analysis. SoftwareX 10, 1-6 (2019)

18. Rosenfeld, A.: Fuzzy digital topology. Information and control 40(1), 76-87 (1979)

19. Rother, C., Kolmogorov, V., Blake, A.: "Grabcut": interactive foreground extraction using iterated graph cuts. TOG 23(3), 309-314 (2004)

20. Salembier, P., Garrido, L.: Binary partition tree as an efficient representation for image processing, segmentation, and information retrieval. TIP 9(4), 561-576 (2000)

21. Udupa, J.K., Saha, P.K., Lotufo, R.A.: Fuzzy connected object definition in images with respect to co-objects. In: Medical Imaging 1999: Image Processing. vol. 3661, pp. 236-245. International Society for Optics and Photonics (1999)

22. Udupa, J.K., Samarasekera, S.: Fuzzy connectedness and object definition: Theory, algorithms, and applications in image segmentation. Graphical models and image processing 58(3), 246-261 (1996)

23. Vachier, C., Meyer, F.: The viscous watershed transform. JMIV 22(2-3), 251-267 (2005)

24. Xu, N., Price, B., Cohen, S., Yang, J., Huang, T.S.: Deep interactive object selection. In: CVPR. pp. 373-381. IEEE (2016)

25. Yin, S., Qian, Y., Gong, M.: Unsupervised hierarchical image segmentation through fuzzy entropy maximization. Pattern Recognition 68, 245-259 (2017) 